\title{
A Systematic Genetic Assessment of ARFGEF2 Mutations in Periventricular Heterotopia
}

\author{
Lina Al Neghery ${ }^{1,2}$, Rosan Kenana ${ }^{1}$, Albandary Al Bakheet ${ }^{1}$, Rawan Al Mass ${ }^{1}$, Faten Al Mutairi, \\ Maysoon Al Sagob ${ }^{1}$, Aliya Qari $^{3}$, Rozeena Huma ${ }^{3}$, Dilek Colak ${ }^{4}$, Maha Daghestani ${ }^{2}$, Namik Kaya ${ }^{1}$, \\ Moeenaldeen Al Sayed ${ }^{3, *}$ \\ ${ }^{1}$ Department of Genetics, King Faisal Specialist Hospital and Research Center, Riyadh, Kingdom of Saudi Arabia \\ ${ }^{2}$ Department of Zoology, College of Science, King Saud University, Riyadh, Kingdom of Saudi Arabia \\ ${ }^{3}$ Department of Medical Genetics, King Faisal Specialist Hospital and Research Center, Riyadh, Kingdom of Saudi Arabia \\ ${ }^{4}$ Department of Biostatistics, Epidemiology and Scientific Computing, King Faisal Specialist Hospital and Research Center, Riyadh, \\ Kingdom of Saudi Arabia
}

Email address:

moeen@kfshrc.edu.sa (M. Al Sayed)

${ }^{*}$ Corresponding author

\section{To cite this article:}

Lina Al Neghery, Rosan Kenana, Albandary Al Bakheet, Rawan Al Mass, Faten Al Mutairi, Maysoon Al Sagob, Aliya Qari, Rozeena Huma, Dilek Colak, Maha Daghestani, Namik Kaya, Moeenaldeen Al Sayed. A Systematic Genetic Assessment of ARFGEF2 Mutations in Periventricular Heterotopia. International Journal of Genetics and Genomics. Vol. 6, No. 1, 2018, pp. 11-17. doi: 10.11648/j.jigg.20180601.13

Received: January 12, 2018; Accepted: January 29, 2018; Published: May 5, 2018

\begin{abstract}
Mutations in ADP-ribosylation factor guanine nucleotide-exchange factor 2 (ARFGEF2) lead to autosomal recessive periventricular heterotopia $(\mathrm{PH})$. To date, 11 mutations, (six missense mutations, one splicing mutation, one small deletion, two small insertions, and one small deletion/insertion) have been reported. Assessing ARFGEF2 mutations will provide a holistic overview of PH. This retrospective study was conducted in 2016 at King Faisal Specialist Hospital and Research Center. For the index patient, magnetic resonance imaging studies revealed a symmetrical focal hyperintensity involving the putamen bilaterally and the inner aspect of the globus pallidus. After family members were genotyped, an autozygosity analysis was performed, followed by exome sequencing of the index patient; A comprehensive filtering approach based on the loss of heterozygosity ( $\mathrm{LOH})$ was used to identify variants in phenotypically relevant genes. We report a consanguineous family with two affected individuals, a boy and a girl, with a history of microcephaly, global developmental delay, intellectual disability, myoclonic seizure, and dystonia. The patients carried a novel nonsense mutation (c.3974G>A, p. Trp1325*) in the Armadillo-type fold domain of $A R F G E F 2$. These findings extend our understanding of the phenotypegenotype correlations for $A R F G E F 2$ mutations.
\end{abstract}

Keywords: Developmental Delay, ARFGEF2, Intellectual Disability, Myoclonic Seizure, Dystonia, Periventricular Heterotopia

\section{Introduction}

The development of the human cerebral cortex is a remarkably complex process. Cortical malfunctions are often the underlying causes of developmental microcephaly, global developmental delay, and intellectual disability, and many such disorders have a genetic basis [1-3]. A failure of neuronal migration from the periventricular region to the developing cortex is a familial inherited disorder known as
PH. The hallmark of this syndrome is the presence of irregular nodules. The nodules of heterotopic gray matter accumulate along the walls of the lateral ventricles. $\mathrm{PH}$ has a wide range of anatomical manifestations, ranging from asymptomatic, small unilateral or bilateral nodules, to extensive agglomerates of heterotopia lining the lateral ventricles. The disease is usually diagnosed by cranial magnetic resonance imaging (MRI) [4-6]. Mutations in $A R F G E F 2$ are associated with a rare autosomal recessive 
form, bilateral periventricular nodular heterotopia (BPNH), with features similar to the described features of $\mathrm{PH}$. $A R F G E F 2$ is located on $20 \mathrm{q} 13.1$ and has 39 exons producing two transcripts that encode BIG2 (brefeldin-A inhibited GEF2 protein), which is involved in vesicular trafficking [7]. ARFGEF2 has highly conserved domains; mutations in these domains might have different phenotypic consequences [8]. In addition to microcephaly and other cognitive disorders, $A R F G E F 2$ is also associated with a phenotype known as "choreadystonic extrapyramidal movement disorder." It has been suggested that ARFGEF2 dysfunction leads to neurotransmitter receptor mislocalization, which causes degeneration in the putamen and basal ganglia. This, in turn, leads to the abnormal movement observed in $\mathrm{PH}$ [9]. Besides mutations in $A R F G E F 2$, earlier studies have also indicated that mutations in the filamin A gene (FLNA) are a frequent genetic cause of $\mathrm{PH}[10]$.

In this study, two individuals with $\mathrm{PH}$ and their family members were studied. The clinical and genetic examinations are presented in detail.

\section{Patients, Materials and Methods}

\subsection{Patients}

In this study, two patients diagnosed with microcephaly, global developmental delay, and intellectual disability were retrospectively evaluated. They were born to parents in a consanguineous Saudi family. The parents were healthy and first cousins. Four female and one male sibling of the affected individuals were reported to be healthy, suggesting a recessive mode of inheritance. Moreover, the high rate in consanguineous marriage in the Saudi population is the main cause in the occurrence of these autosomal recessive syndromes [11-13].

The index case was a girl. A normal pregnancy and delivery were reported. She had jaundice after birth, for which she received phototherapy for 24 hours. Her development in all domains was reported by her family to be normal until 7 months of age. At 7 months, she had an intercurrent febrile, possibly viral illness that was associated with generalized tonic-clonic seizures. She recovered from the febrile episode, but failed to reach any meaningful developmental milestones thereafter. Her clinical course was then marked by microcephaly, global developmental delay, intellectual disability, myoclonic seizure, and dystonia. She was referred for genetic evaluation and was first genetically examined at the age of 3 years. At this age, failure to thrive was noted, with a weight and height far below the $3^{\text {rd }}$ percentile for this age. She had microcephaly, complete head lag, axial hypotonia, spasticity in all extremities, myoclonic seizures, abnormal dystonic movement of both hands, and no facial dysmorphism. The patient was visually not attentive. An eye examination including the fundus was normal. An abdominal examination did not reveal organomegaly. Visual evoked potential (VEP) showed a normal flash VEP, indicating that visual inputs reached the cortex. A metabolic workup, including acylcarnitines, plasma amino acids, and urine organic acid level analyses, was normal. Lactate level was slightly elevated, i.e., $2.7 \mu \mathrm{mol} / 1$ (normal, up to 2); however, it was normal in a repeat analysis. Urine excretion levels of creatine, guanidine acetoacetic acid, and creatinine were within the respective reference ranges. Normal fibroblast activity of palmitoyl protein thioesterase I and tripeptidyl-peptidase I ruled out neuronal ceroid lipofuscinosis 1 and 2, respectively. Respiratory chain complex activity was also normal in the cultured fibroblasts of the patients.

Brain MRI revealed a symmetric focal hyperintensity involving the bilateral putamen and the inner aspect of the globus pallidus, suggesting a possible mitochondrial disorder. The thalami were unaffected; the caudate nuclei were normal, and the white matter showed no abnormality. The corpus callosum was normal, and no restricted diffusion was observed. The pituitary gland also was normal in size. Observed orbital structures were within normal limits. MR spectroscopy did not show any abnormal metabolites levels. $\mathrm{N}$-Acetyl aspartate, choline, and creatinine levels were within normal limits.

Using autozygosity mapping coupled with whole exome sequencing, a variant resulting in the truncation of the $A R F G E F 2$ gene, which is associated with the phenotype, was identified.

Her younger brother similarly presented with a normal perinatal history and cessation of further developmental milestones by 9 months of age. Like his sister, salient features of his illness included microcephaly, global developmental delay, intellectual disability, myoclonic seizures, and abnormal dystonic movements of the hands, axial hypotonia, and spasticity in all extremities. A targeted analysis of $A R F G E F 2$ confirmed the same variant as that detected in his older sister.

\subsection{Ethical Approval}

The study was performed in 2016 at King Faisal Specialist Hospital and Research Center in accordance with institutional review board approved protocols (IRB-AP) (RAC \#2060035). Written informed consent was obtained from all the participants, as per the IRB-AP. One consanguineous family with two affected siblings who showed microcephaly, global developmental delay, intellectual disability, myoclonic seizure, and dystonia was studied.

\subsection{Sample Collection}

Blood samples were obtained according to standard protocols. DNA was extracted using a commercially available kit from Qiagen (Venlo, Netherlands). Nucleic acid quality and quantity were determined using the NanoDrop ND-1000 and the Agilent Bioanalyzer.

\subsection{Affymetrix Axiom Human Mapping Assays}

Axiom arrays (Affymetrix, Santa Clara, CA, USA) were used for genome-wide SNP genotyping using the Axiom $\mathbb{}$ 
2.0 Assay. Gene Titan Array plate registration, target preparation, labeling, hybridization, scanning, image processing, and preliminary data analyses were all performed according to the manufacturer's protocols and guidelines.

\subsection{Autozygosity Detection and Homozygosity Mapping}

Runs of homozygosity (ROH) blocks using Axiom's SNP genotyping calls were determined by LOH detection, as determined using AutoSNPa with the default settings [10]. The autozygosity analysis was performed using previously established protocols [14-18]. Briefly, SNPs indicating LOH over 2 megabases were considered a major block and a targeted region of interest.

\subsection{Whole Exome Sequencing}

Whole exome sequencing (WES) was performed using the Agilent Genomics SureSelect system (Santa Clara, CA, USA). Sequences were read using the HiSeq 2000 (Illumina, San Diego, CA, USA) with a minimum of 30 -fold coverage. Data were analyzed using publicly and commercially available software and tools.

\subsection{Confirmation by Targeted Sequencing}

DNA was amplified using $A R F G E F 2$-specific primers in separate reactions. Each fragment was sequenced using one of the PCR primers, according to standard protocols.

\subsection{Bioinformatics Analyses}

Several publicly available sequence-variant databases, such as ClinVar, as well as commercially available software, such as HGMD Professional from BIOBASE (Qiagen), were searched. The variant was also absent in dbSNP, Ensembl, 1000 Genomes Project, the Exome Aggregation Consortium (ExAC), the National Heart, Lung, and Blood Institute Exome Variant Server (EVS), and the Genome Aggregation Database (gnomAD).

\section{Results}

After a thorough clinical evaluation, the family members were genotyped using Affymetrix genome-wide SNP arrays, which contain more than 600,000 well-documented SNPs. The genotypes generated from these SNP calls were evaluated. Clinical ROH blocks of any size are useful for the diagnosis of an autosomal recessive disorder by autozygosity mapping (Figure 1) [19, 20]. In addition to, it has been suggested that the candidate genes should be contained within a $2 \mathrm{Mb}$ interval [21].

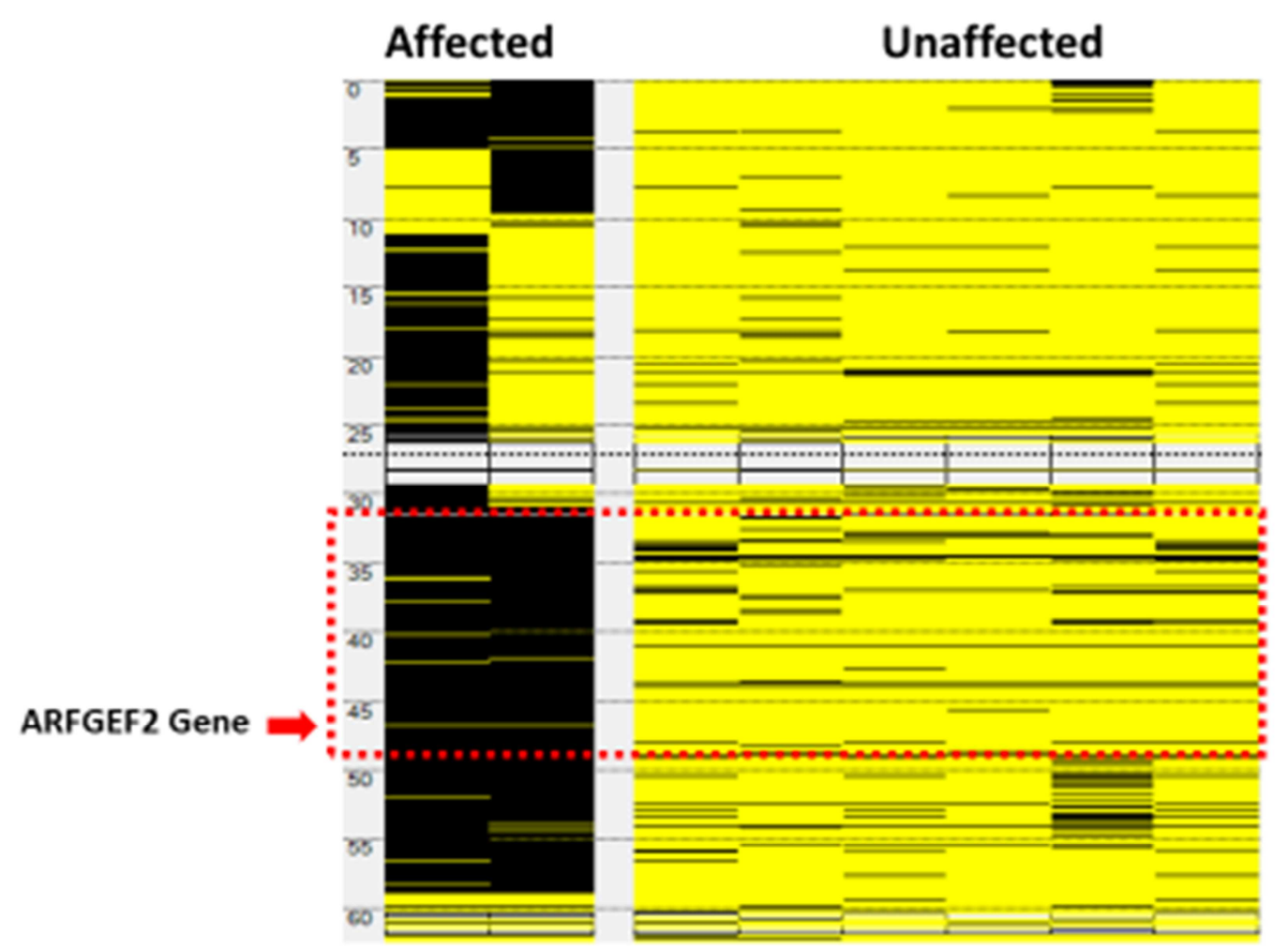

Figure 1. The shared regions of homozygosity in the family contains 150 genes, including the ARFGEF2 gene.

As part of the diagnostic procedure, the DNA of the index patient was sent for exome sequencing. The raw data were obtained and analyzed locally. A comprehensive filtering analysis of the WES variants was performed in a step-by-step manner. The parents are closely related, and the pattern of the disease is consistent with an autosomal recessive mode of inheritance; therefore, the initial stages of the filtering process focused on homozygosity-related approaches. The first step involved overlying the autozygome (specific to the patients) on the WES variants. This approach helped to 
minimize unexplained genetic variance. The second step included the detection of homozygous variants, especially those expected to have lethal consequences, such as truncation mutations. The next step involved an analysis of homozygous variants found in phenotypically relevant genes, such as $A R F G E F 2$. Indeed, p. Trp1325* in ARFGEF2 was the only candidate identified using these filtering steps. We then performed confirmatory Sanger sequencing of the

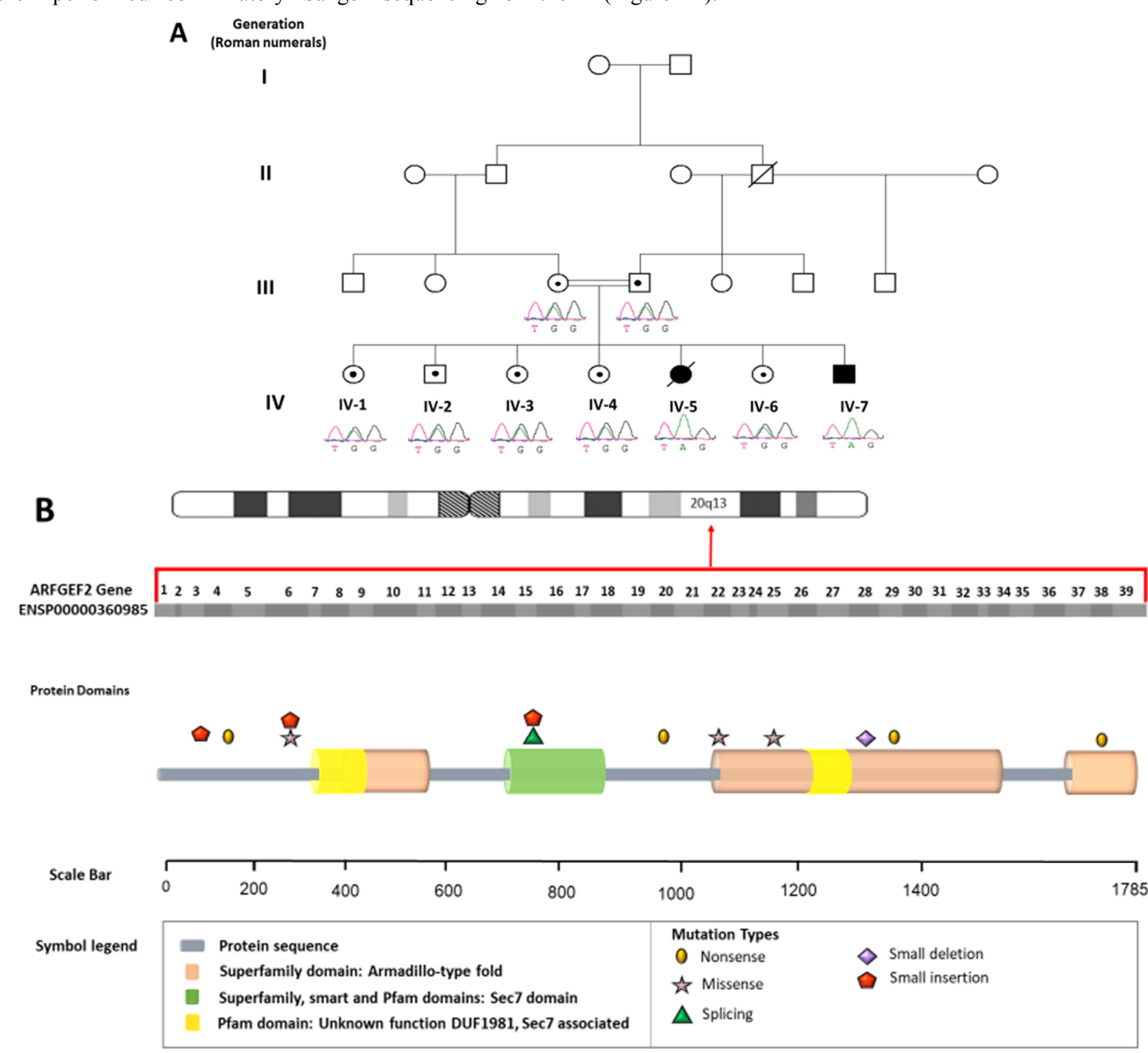

Figure 2. c.3974G>A, p. Trp1325* mutation identified in the family.

(A) Pedigree of the consanguineous Saudi family with periventricular heterotopia showed the segregation of a mutation in the ARFGEF1 gene within this family. Squares represent males and circles represent females. A filled symbol refers to the affected members in the family. Black symbols denote affected individuals; diagonal lines denote deceased individuals; double bars denote consanguinity. Dots represent individuals who carried the mutation.

(B) Overview of the ARFGEF2 gene. (1) Diagram variant. In particular, a sequence analysis of $A R F G E F 2$ was performed using the reference sequence NM_006420.2, according to de Wit et al. [9]. This DNA alteration has not yet been described in the literature. The verified sequence variant was attached to the family pedigree (Figure 2A). This variant leads to a premature stop codon (c.3974G $>$ A; p. Trp1325*) that results in a truncated Armadillo-type fold protein domain (Figure 2B). 


\section{Discussion}

ARFs require assistance proteins, referred to as guaninenucleotide-exchange factors (GEFs), to catalyze the exchange of GDP for GTP in the formation of coated vesicles from the Golgi apparatus, trans-Golgi network, endosomes, lysosomes, and the plasma membrane. Intracellular vesicle trafficking pathways recycle and transport a specific subgroup of membrane proteins, including sorting receptors and many transporters. Trafficking is very important for cell proliferation and migration during cerebral cortical development in humans [22].

Studies have suggested an association between a group of disorders and the failure of vesicle trafficking pathways, which, in turn, leads to a failure to transport proteins to the cell membrane. This transportation failure has been demonstrated for the GABA (A) receptor and other cell surface receptors [23]. The ARFGEF2 protein, also known as $\mathrm{BIG} 2$, is expressed at a high level in the brain during neuron proliferation and migration [24]. RNA interference to knock down BIG2 expression showed that the inhibition of BIG2 decreases cell proliferation, and this likely causes dysfunctional Golgi organization and endosome recycling. The disruption in endosome recycling subsequently causes problems in neuronal motility $[25,26]$. Moreover, the neuroependymal lining was observed after the disruption of BIG2 in mice by brefeldin-A (BFA) treatment. Finally, the disruption of BIG2 not only causes motility problems, but also affects the integrity of cell morphology and cell-cell integrity along the neuroependymal lining, leading to periventricular nodule formation in mice [25].

Citterio and colleagues observed that markers of stress in the endoplasmic reticulum, part of the cell network along with Golgi organization, would increase susceptibility to apoptosis in specific neurons, which also might play a role in the development of PH [27]. Another study suggested that the in vitro treated neurons by BAF has inhibitory effects on BIG2 which interfere with the normal localization of an intracellular molecules such as the E-cadherin and its cytoplasmic ligand, B-catenin, on neurons, which restricts their localization to within the Golgi apparatus, i.e., it prevents their transport to the cell surface, these finding emphasized an importance of the proliferation of neural precursor in brain size and the properly migration in the intact ventricular zones [7, 28, 29].

The results of these previous studies suggested that there is a relationship between the phenotype of our patient and the loss of ARFGEF2 function. Our analysis revealed a novel previously unreported homozygous nonsense (c.3974G $>$ A, p. Trp1325*) variant in exon 29 of the $A R F G E F 2$ gene. In addition to our case, additional cases of $\mathrm{PH}$ associated with mutations in ARFGEF2 have been reported in other studies [7, 9, 23, 30]. Characteristics of $A R F G E F 2$ mutations and related protein domains, including the mutation presented in this study (Table 1).

Table 1. Summary of the ARFGEF2 mutations and related protein domains.

\begin{tabular}{|c|c|c|c|c|c|}
\hline $\mathbf{N}$ & Database & cDNA & Exon & Protein & Mutation Type \\
\hline 1 & HGMD & c. $388 \mathrm{C}>\mathrm{T}$ & 4 & p. $\left(\mathrm{G} \ln 130^{*}\right)$ & Nonsense \\
\hline 2 & HGMD & c. $625 \mathrm{G}>\mathrm{A}$ & 6 & p. (Glu209Lys) & Missense \\
\hline 3 & HGMD & c. $2776 \mathrm{C}>\mathrm{T}$ & 20 & p. $\left(\operatorname{Arg} 926^{*}\right)$ & Nonsense \\
\hline 4 & HGMD & c. $3022 \mathrm{G}>\mathrm{C}$ & 22 & p. (Gly1008Arg) & Missense \\
\hline 5 & HGMD & c. $3326 \mathrm{G}>\mathrm{A}$ & 25 & p.(Arg1109His) & Missense \\
\hline 6 & HGMD & c. $5126 \mathrm{G}>\mathrm{A}$ & 38 & p. $\left(\operatorname{Trp} 1709^{*}\right)$ & Nonsense \\
\hline 7 & HGMD & c. $1958+1 \mathrm{G}>\mathrm{A}$ & 15 & - & Splicing \\
\hline 9 & HGMD & c.656dupC & 6 & p.(Val220Cysfs*35) & Small insertion \\
\hline 10 & HGMD & c.2031_2038dupCATAGCCC & 15 & p.(Gln680Profs*2) & Small insertion \\
\hline 11 & HGMD & c. $242 \_\overline{2} 49$ delCAAGGGTAinsAAAGGTT & 3 & p.(Pro81Glnfs*34) & Small insertion \\
\hline 12 & & c. $3974 \mathrm{G}>\mathrm{A}$ & 29 & p. $(\operatorname{Trp} 1325 *)$ & Nonsense \\
\hline
\end{tabular}

Table 1. Continued.

\begin{tabular}{|c|c|c|c|c|}
\hline $\mathbf{N}$ & Mt Location on Domains & Phenotype & Ethnicity & References \\
\hline 1 & - & Periventricular nodular heterotopia with dystonia & Turkish & Bardón Cancho (2014) \\
\hline 2 & - & Periventricular nodular heterotopia with dystonia & Turkish & Sheen (2004) \\
\hline 3 & - & Periventricular nodular heterotopia with dystonia & Unknown & Makrythanasis (2014) \\
\hline 4 & Armadillo-type fold & Landau Kleffnersyndrome & Irish and Australian & Conroy (2014) \\
\hline 5 & Armadillo-type fold & Diaphragmatic hernia, congenita & Unknown & Yu (2015) \\
\hline 6 & Armadillo-type fold & $\begin{array}{l}\text { Movement disorder, developmental delay, } \\
\text { microcephaly and cardiomyopathy }\end{array}$ & Turkish & Yilmaz (2015) \\
\hline 7 & Sec7 domain & $\begin{array}{l}\text { West syndrome, microcephaly, periventricular heterotopia, small } \\
\text { corpus callosum \& psychomotor retardation }\end{array}$ & Palestinian & Banne (2013) \\
\hline 8 & Armadillo-type fold & Choreadystonic movement disorder and Periventricular heterotopia & Dutch & de Wit (2009) \\
\hline 9 & - & Global developmental delay, epilepsy and hydrocephalus & Saudi & Alazami (2015) \\
\hline 10 & Sec7 domain & Choreadystonic movement disorder and periventricular & Dutch & de Wit (2009) \\
\hline 11 & - & Periventricular heterotopia with microcephaly & $\begin{array}{l}\text { Mouse } \\
\text { Brussels }\end{array}$ & $\begin{array}{l}\text { Sheen }(2004) \\
\text { Tanyalçin (2013) }\end{array}$ \\
\hline 12 & Armadillo-type fold & $\begin{array}{l}\text { An Autosomal-Recessive Periventricular Bilateral Nodular } \\
\text { Heterotopia with Microcephaly, Global development delay and } \\
\text { Intellectual disability }\end{array}$ & Saudi & 2017 \\
\hline
\end{tabular}


Previous studies have focused on the Sec7 domain, which acts on the surface of membranes to insert another regulatory protein (called Arf1) into the membranes of the Golgi complex [31, 32]. Hence, it is necessary to reconsider the importance of the Armadillo-type fold domain.

\section{Conclusion}

A mutation in ARFGEF2 must be considered in the presence of BPNH and putamen hyperintensity in children exhibiting microcephaly, global developmental delay, seizures, and intellectual disability, especially in cases with a lethal outcome.

\section{Acknowledgements}

We are grateful to the patients and the family members for their participation in our study. This work was conducted through intramural funds given to Dr. Namik Kaya and Dr. Moeen aldeen AlSayed by King Faisal Specialist Hospital and Research Centre (KFSHRC- RAC: 2060035). We would also like to thank the National Plan for Science, Technology and Innovation program under King Abdulaziz City for Science and Technology (NSTIP/KACST) for supporting Dr. Namik Kaya (11-BIO2221-20) and Dr. Dilek Colak (11BIO2072-20).

\section{Conflict of Interest}

The authors do not have any commercial associations that influenced the design or presentation of the study or that might pose or create a conflict of interest with the information presented in the manuscript. Thus, we have no conflict of interest to disclose.

\section{References}

[1] Kamuro K, Tenokuchi Y. Familial periventricular nodular heterotopia. Brain Dev 1993; 15: 237-241.

[2] Wang R, Khan A, Han S, Zhang X. Molecular analysis of 23 Pakistani families with autosomal recessive primary microcephaly using targeted next-generation sequencing. J Hum Genet 2017; 62(2):299-304.

[3] Wolfe K, Stueber K, McQuillin A, Jichi F, Patch C, Flinter F, et al. Genetic testing in intellectual disability psychiatry: Opinions and practices of UK child and intellectual disability psychiatrists. J Appl Res Intellect Disabil 2017; 1-12.

[4] Guerrini R, Carrozzo R. Epileptogenic brain malformations: clinical presentation, malformative patterns and indications for genetic testing. Seizure 2001; 10: 532-543; quiz 44-47.

[5] Sidman RL, Rakic P. Neuronal migration, with special reference to developing human brain: a review. Brain Res $1973 ; 62: 1-35$.

[6] Rakic P. Cell migration and neuronal ectoplasm in the brain. Birth Defects Orig Artic Ser 1975; 1975: 95.
[7] Sheen VL, Ganesh VS, Topcu M, Sebire G, Bodell A, Hill RS, et al. Mutations in ARFGEF2 implicate vesicle trafficking in neural progenitor proliferation and migration in the human cerebral cortex. Nature Genet 2004; 36: 69-76.

[8] Sheen VL. Filamin A and Big2: a shared endocytic pathway. Bioarchitecture 2014; 4: 53-57.

[9] de Wit MC, de Coo IF, Halley DJ, Lequin MH, Mancini GM. Movement disorder and neuronal migration disorder due to ARFGEF2 mutation. Neurogenetics 2009; 10: 333336 .

[10] Parrini E, Ramazzotti A, Dobyns WB, Mei D, Moro F, Veggiotti $\mathrm{P}$, et al. Periventricular heterotopia: phenotypic heterogeneity and correlation with Filamin A mutations. Brain 2006; 129: 1892-1906.

[11] Alfares AA, Alfadhel M, Wani T, Alsahli S, Alluhaydan I, Al Mutairi $\mathrm{F}$, et al. A multicenter clinical exome study in unselected cohorts from a consanguineous population of Saudi Arabia demonstrated a high diagnostic yield. Mol Genet Metab 2017;121 (2):91-95.

[12] Yavarna T, Al-Dewik N, Al-Mureikhi M, Ali R, Al-Mesaifri F, Mahmoud L, et al. High diagnostic yield of clinical exome sequencing in Middle Eastern patients with Mendelian disorders. Hum Genet 2015; 134:967-980.

[13] Anazi S, Maddirevula S, Faqeih E, Alsedairy H, Alzahrani F, Shamseldin $\mathrm{H}$, et al. Clinical genomics expands the morbid genome of intellectual disability and offers a high diagnostic yield. Mol Psychiatry 2017; 22(4):615-624.

[14] Al-Hassnan ZN, Al-Dosary M, Alfadhel M, Faqeih EA, Alsagob M, Kenana R, et al. ISCA2 mutation causes infantile neurodegenerative mitochondrial disorder. J Med Genet 2015; 52: 186-194.

[15] Al-Owain M, Colak D, Al-Bakheet A, Al-Hashmi N, Shuaib T, Al-Hemidan A, et al. Novel mutation in GLRB in a large family with hereditary hyperekplexia. Clin Genet 2012; 81: 479-484.

[16] Al-Sayed MD, Al-Zaidan H, Albakheet A, Hakami H, Kenana $\mathrm{R}$, Al-Yafee $\mathrm{Y}$, et al. Mutations in NALCN cause an autosomal-recessive syndrome with severe hypotonia, speech impairment, and cognitive delay. Am J Hum Genet 2013; 93: 721-726.

[17] Kaya N, Aldhalaan H, Al-Younes B, Colak D, Shuaib T, AlMohaileb F, et al. Phenotypical spectrum of cerebellar ataxia associated with a novel mutation in the CA8 gene, encoding carbonic anhydrase (CA) VIII. Am J Med Genet B Neuropsychiatr Genet 2011; 156B: 826-834.

[18] Kaya N, Alsagob M, D'Adamo MC, Al-Bakheet A, Hasan S, Muccioli M, et al. KCNA4 deficiency leads to a syndrome of abnormal striatum, congenital cataract and intellectual disability. J Med Genet 2016.

[19] Alkuraya FS. Homozygosity mapping: one more tool in the clinical geneticist's toolbox. Genet Med 2010; 12: 236-239.

[20] Sund KL, Zimmerman SL, Thomas C, Mitchell AL, Prada CE, Grote L, et al. Regions of homozygosity identified by SNP microarray analysis aid in the diagnosis of autosomal recessive disease and incidentally detect parental blood relationships. Genet Med 2013; 15: 70-78. 
[21] Alkuraya FS. Discovery of rare homozygous mutations from studies of consanguineous pedigrees. Curr Protoc Hum Genet 2012; 75:1-13.

[22] Chia PZ, Gunn P, Gleeson PA. Cargo trafficking between endosomes and the trans-Golgi network. Histochem Cell Biol 2013; 140: 307-315.

[23] Banne E, Atawneh O, Henneke M, Brockmann K, Gartner J, Elpeleg $\mathrm{O}$, et al. West syndrome, microcephaly, grey matter heterotopia and hypoplasia of corpus callosum due to a novel ARFGEF2 mutation. J. Med. Genet 2013; 50: 772-775.

[24] Chen ZW, Olsen RW. GABAA receptor associated proteins: a key factor regulating GABAA receptor function. J Neurochem 2007; 100: 279-294.

[25] Ferland RJ, Batiz LF, Neal J, Lian G, Bundock E, Lu J, et al. Disruption of neural progenitors along the ventricular and subventricular zones in periventricular heterotopia. Hum Mol Genet 2008; 18: 497-516.

[26] Ishizaki R, Shin HW, Mitsuhashi H, Nakayama K. Redundant roles of BIG2 and BIG1, guanine-nucleotide exchange factors for ADP-ribosylation factors in membrane traffic between the trans-Golgi network and endosomes. Mol Biol Cell 2008; 19: 2650-2660.
[27] Citterio C, Vichi A, Pacheco-Rodriguez G, Aponte AM, Moss J, Vaughan M. Unfolded protein response and cell death after depletion of brefeldin A-inhibited guanine nucleotideexchange protein GBF1. Proc Natl Acad Sci USA 2008; 105: $2877-2882$.

[28] Chenn A, Walsh CA. Regulation of cerebral cortical size by control of cell cycle exit in neural precursors. Science 2002; 297: 365-369.

[29] Klymkowsky MW, Parr B. The body language of cells: the intimate connection between cell adhesion and behavior. Cell 1995; 83: 5-8.

[30] Tanyalcin I, Verhelst H, Halley DJ, Vanderhasselt T, Villard L, Goizet $\mathrm{C}$, et al. Elaborating the phenotypic spectrum associated with mutations in $A R F G E F 2$ : case study and literature review. Eur J Paediatr Neurol 2013; 17: 666-670.

[31] Richardson BC, Halaby SL, Gustafson MA, Fromme JC. The Sec7 N-terminal regulatory domains facilitate membraneproximal activation of the Arfl GTPase. eLife 2016; 5: 1-20.

[32] Jackson CL. Mechanisms of transport through the Golgi complex. J Cell Sci 2009; 122: 443-452. 